\title{
Status of the HMPID CsI-RICH project for ALICE at the CERN/LHC
}

\author{
A. Di Mauro \\ on behalf of the ALICE/HMPID group
}

\begin{abstract}
The ALICE High Momentum Particle Identification (HMPID) detector, presently under construction, consists of seven identical proximity focusing RICH counters, covering in total $11 \mathrm{~m}^{2}$ and exploiting large area CsI photocathodes for Cherenkov light imaging. The detector design, assembly and quality checks will be presented, with particular emphasis on the CsI photocathodes production and validation procedure. Performance studies in beam tests of the produced detector modules and results on ageing of CsI photocathodes due to ions bombardment will be discussed.
\end{abstract}

\section{INTRODUCTION}

ALICE (A Large Ion Collider Experiment) is a general purpose heavy-ion experiment, specifically optimized to study $\mathrm{Pb}-\mathrm{Pb}$ collisions at the CERN/LHC, at a center of mass energy per nucleon pair of $\sqrt{\mathrm{S}_{\mathrm{NN}}}=5.5 \mathrm{TeV}$ with a luminosity of $10^{27}$ $\mathrm{cm}^{-2} \mathrm{~s}^{-1}[1]$. Head-on collisions between ultra-relativistic heavy ions are expected to provide the conditions of energy densities corresponding to the phase transition of hadronic matter to a short-lived state where quark are no longer confined within the nucleus (Quark Gluon Plasma). Interactions between lower mass nuclei and p-p will also be studied as benchmark and reference data for heavy-ion physics. ALICE will address both hadronic and leptonic signals in a wide momentum range, from $100 \mathrm{MeV} / \mathrm{c}$ to $100 \mathrm{GeV} / \mathrm{c}$, up to the largest anticipated charged particle density of 8000 per unit of rapidity in central $\mathrm{Pb}-\mathrm{Pb}$ collisions. Particle identification (PID) plays a key role in the characterization of such events and a complex PID scheme has been implemented, with three detectors covering the full ALICE barrel - the Inner Tracking System (ITS), the TPC and the Time-of-Flight (TOF) - and a single-arm detector, the High Momentum PID (HMPID) devoted to the identification of high transverse momentum $p_{\mathrm{T}}$ particles, i.e. pions, kaons and protons in the range from 1 to $5 \mathrm{GeV} / \mathrm{c}$.

Manuscript received November 1, 2004

A. Di Mauro is with CERN, the Eurpoean Laboratory for Particle Physics, 1211 Genève 23, Switzerland (e-mail: antonio.di.mauro@cern.ch, telephone: +41-227676612).
The HMPID system consists of an array of seven identical RICH (Ring Imaging Cherenkov) detector modules of $1.3 \mathrm{~m} \times$ $1.3 \mathrm{~m}$, arranged in a cupola-like structure and located at a radial distance of $4.7 \mathrm{~m}$ from the interaction point (Fig.1) [2]. Each module is equipped with six CsI photocathodes (PCs) of $0.64 \mathrm{~m} \times 0.4 \mathrm{~m}$, thus resulting in a total active area of $11 \mathrm{~m}^{2}$. The detector has been designed to identify pions and kaons in the range $1 \leq \mathrm{p}_{\mathrm{T}} \leq 3 \mathrm{GeV} / \mathrm{c}$ and protons in the range $2 \leq \mathrm{p}_{\mathrm{T}} \leq 5$ $\mathrm{GeV} / \mathrm{c}$, with the largest anticipated charged particles multiplicity of $100 \mathrm{~m}^{-2}$.

Like all detectors involved in LHC experiments, the HMPID system is presently under construction. In this paper we will review some aspects of the detector design, assembly and validation, in particular for the CsI PCs that constitute the most essential parts of our system.

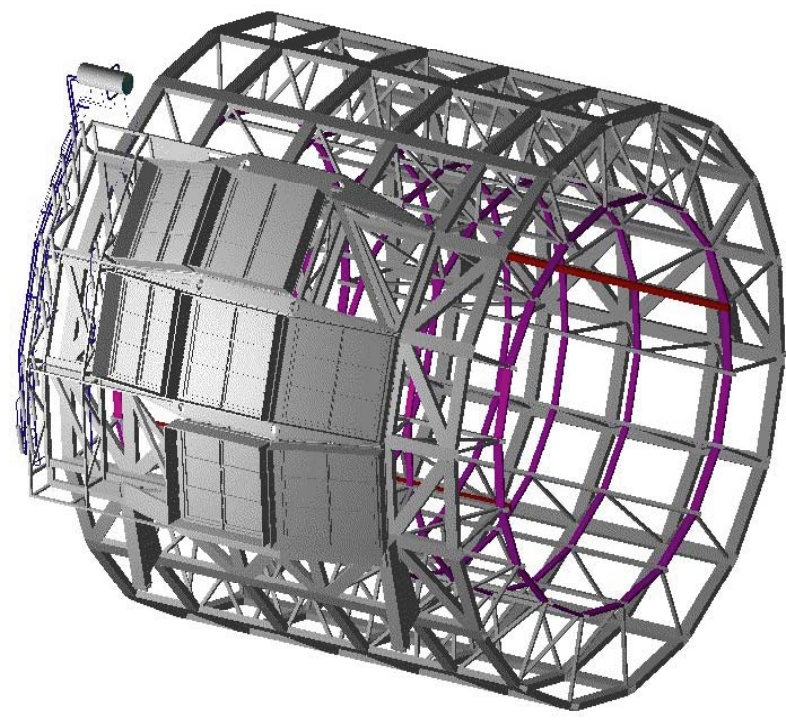

Fig. 1. View of the ALICE space frame. The seven HMPID modules are mounted onto a cradle support; each module is oriented in a way to minimize the dip angle of incoming particles within $10^{\circ}$ around the perpendicular. 


\section{DETECTOR LAYOUT}

The HMPID RICH detector has a proximity focusing configuration, with a $1.5 \mathrm{~cm}$ thick layer of $\mathrm{C}_{6} \mathrm{~F}_{14}$ (perfluorohexane) liquid radiator circulated in vessels, with $5 \mathrm{~mm}$ fused silica exit windows, transparent to the Cherenkov radiation (Fig. 2). The $\mathrm{C}_{6} \mathrm{~F}_{14}$ refractive index at $\lambda=175 \mathrm{~nm}$ is $\mathrm{n}=1.2989$, corresponding to $\beta_{\min }=0.77$, namely a threshold momentum $\mathrm{p}_{\mathrm{th}}=$ $1.21 \mathrm{~m} \mathrm{GeV} / \mathrm{c}$, with $m$ equal to the particle mass in $\mathrm{GeV} / \mathrm{c}^{2}$. The photon detector is an open geometry MWPC operated with $\mathrm{CH}_{4}$ at atmospheric pressure; it has one cathode plane segmented into $80 \times 48$ pads of $8 \mathrm{~mm} \times 8.4 \mathrm{~mm}$ and coated with a $300 \mathrm{~nm}$ photosensitive layer of CsI. An additional wire plane positively biased, located just after the quartz window, acts as collection electrode to prevent electrons released by charged particles in the proximity gap entering the $4 \mathrm{~mm}$ sensitive gap.

Multiplexed analogue pad readout allows event reconstruction by measurement of the centroid. Two ASICs have been specifically developed for this application: the GASSIPLEX [3], a 16-channels charge sensitive pre-amplifier and shaper with $1.2 \mu$ s peaking time and $1000 \mathrm{e}^{-}$noise measured on detector, and the DILOGIC, a digital processor for zero suppression.

\section{STATUS OF THE PRODUCTION}

The HMPID modules production is divided into four main lines: the MWPC, the radiator vessels, the CsI PC, the FrontEnd electronics (FEE).

Five out of seven chambers including the MWPC and the radiator vessels have been completed.

The mass production of 42 CsI PCs started in May '04, 12 have been used to equip Module- 2 and Module-3 in testbeam.

For the FEE, all the chips needed to readout the 161280 pads, 10080 GASSIPLEX and 3360 DILOGIC, have been mounted on cards and tested.

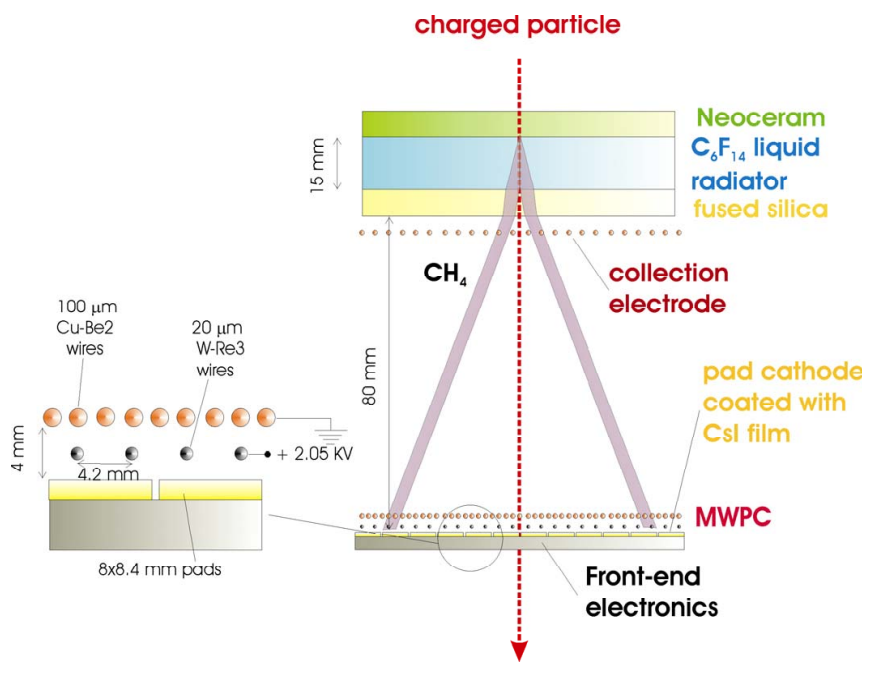

Fig. 2. Schematic cross-section of the HMPID RICH detector.

\section{DETECTOR ASSEMBLY AND QUALITY CHECKS}

Each module is equipped with three radiator vessels of 1330 $\mathrm{mm} \times 413 \mathrm{~mm} \times 24 \mathrm{~mm}$, made of NEOCERAM $^{\circledR}$, a glass material having thermal coefficient very close to the fused silica plates used as UV-transparent windows (Fig. 3). Three rows of ten fused silica rods are glued between the NEOCERAM bottom plate and the quartz windows to achieve the necessary strength to withstand the hydrostatic load. The vessels are He-leak tested twice, after the gluing and after the mounting on the support panel. Such a panel is a composite plate obtained by sandwiching two $0.5 \mathrm{~mm} \mathrm{Al}$ foils with a 50 mm plate of ROHACELL- $51^{\circledR}$, a very stiff and light material $\left(0.0513 \mathrm{~g} / \mathrm{cm}^{3}\right)$, to minimize the radiation length of the detector "entrance window".

The MWPC is a stack of four Al frames of $1.3 \mathrm{~m} \times 1.3 \mathrm{~m}$, holding the different wire planes, machined with a flatness of $0.1 \mathrm{~mm}$; an accuracy of $50 \mu \mathrm{m}$ is achieved in the sensitive gap and in the positioning of essential elements. Such a stack is closed on one side by the radiator panel support and on the other side by the CsI PCs; FKM 75 o-rings are inserted between the frames to form a leak tight vessel. Usual quality checks are performed during and after the assembly: metrology, wire tension measurement, leak rate measurement, $\mathrm{HV}$ commissioning under $\mathrm{CO}_{2}$ and $\mathrm{CH}_{4}$, gain mapping with a Sr-90 source.

\section{CSI PC PRODUCTION AND VALIDATION}

A pad cathode plane is assembled by gluing two $\mathrm{Cu}$-clad multilayer PCBs onto an $\mathrm{Al}$ frame using a vacuum table to achieve a flatness better than $50 \mu \mathrm{m}$ (Fig. 4).

The $\mathrm{Cu}$ pads are covered with a $7 \mu \mathrm{m}$ layer of $\mathrm{Ni}$ and a 0.5 $\mu \mathrm{m}$ of $\mathrm{Au}$, the first acting as a barrier preventing the reaction of CsI with $\mathrm{Cu}$, the second being an optimal substrate for CsI coating. The substrate preparation is crucial to achieve a high and reproducible CsI quantum efficiency (QE) and a special procedure has been established to ensure the necessary surface quality [4].

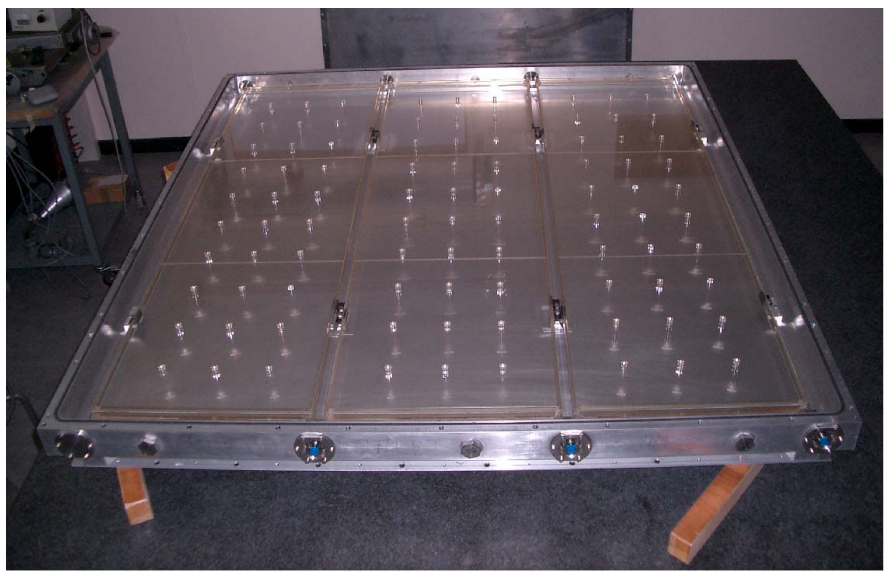

Fig. 3. The three radiator vessels equipping one HMPID module. 
The pad cathode is coated in a large evaporation plant, where CsI is evaporated by Joule effect from four molybdenum crucibles at a pressure of $5 \times 10^{-7}$ mbar. After the CsI deposition, heat conditioning under vacuum at $60{ }^{\circ} \mathrm{C}$ for $12 \mathrm{~h}$ is performed to achieve the final QE. Finally, since CsI is very hygroscopic, the $\mathrm{PC}$ is encapsulated under $\mathrm{Ar}$ in a protective box and always kept under gas flow to avoid any exposure to moisture, until it is mounted on the detector by means of a large glove-box that can be attached to the HMPID module.

The CsI QE is essential for the PID performance, which depends on the number of detected Cherenkov photons per ring (Fig. 5). Given the unavoidable variations of the $\mathrm{PC} \mathrm{QE}$ in the mass production, we established a validation procedure using two available tools: the VUV scanner and the testbeam.

The VUV scanner provides a mapping of the integral illumination response of the produced PC [5]. Although useful for event analysis, the integral response mapping cannot be used alone to set a threshold for acceptance and has to be put in relation with the performance measured in testbeam Cherenkov events, from which the differential QE can be estimated [6].

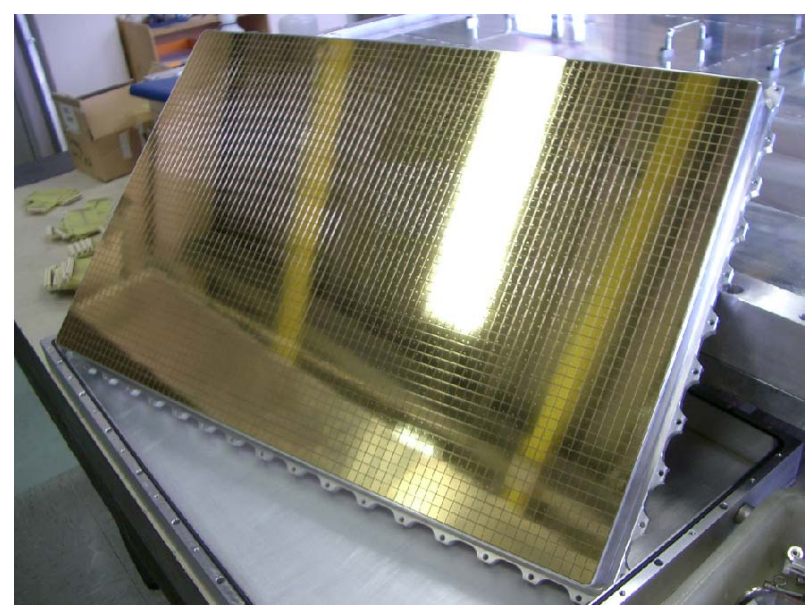

Fig. 4. A pad cathode board $(80 \times 48$ pads of $8 \mathrm{~mm} \times 8.4 \mathrm{~mm})$ before the coating with CsI.

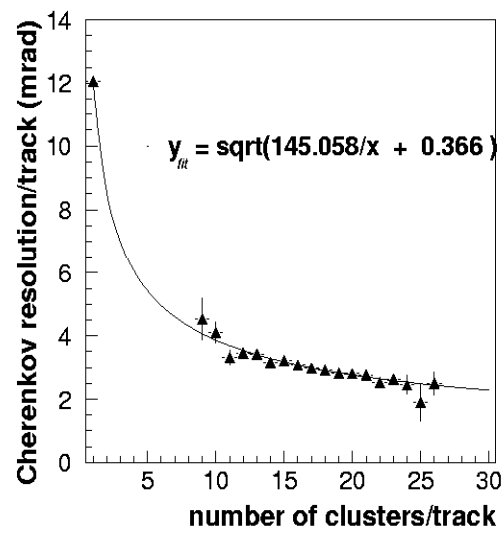

Fig. 5. Measured Cherenkov angle resolution/track as a function of the number of clusters/track. The requirements in terms of a given resolution for $\pi / \mathrm{k}$ identification (e.g. $3 \mathrm{mrad}$ for $5 \sigma$ separation) translate into a threshold on the number of clusters (15 per track).

\section{THE VUV SCANNER}

The VUV scanner system is implemented in a large vessel attached to the evaporation plant. After CsI deposition a PC can be translated to the scanner system where the photocurrent induced by a collimated light beam from a deuterium lamp with $\mathrm{MgF}_{2}$ window, normalized to a reference $\mathrm{PM}$, is recorded over the full photosensitive area. Fig. 6 shows the normalized photocurrent mapping measured on the first $\mathrm{PC}$ of the series production, $\mathrm{PC} 41$, characterized by an acceptable QE inhomogeneity of $5 \%$. A summary of the average response of the first 13 produced CsI PCs is reported in Fig.7.

\section{MODULES COMMISSIONING IN TESTBEAM}

The commissioning in testbeam is a fundamental step of the validation procedure both for the MWPC and for the PCs of each module. The MWPC is studied in terms of operation stability and gain uniformity, while the CsI PCs QE is estimated from testbeam data with the support of a MonteCarlo program [6].

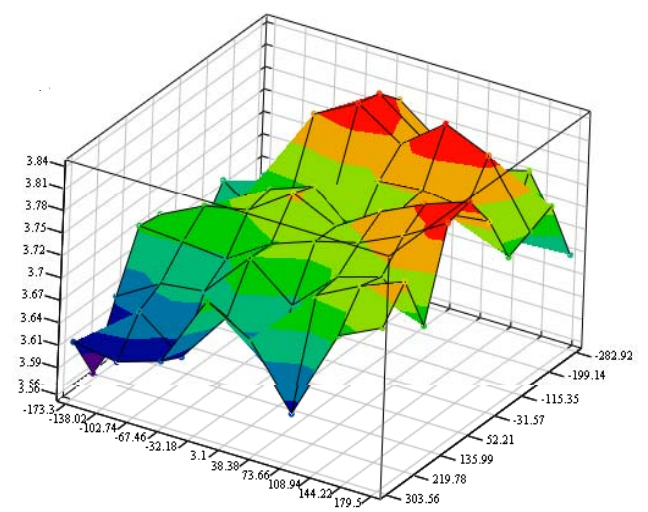

Fig. 6. The normalized photocurrent response mapping of PC41 characterized by an average of 3.72 over the full scanned area.

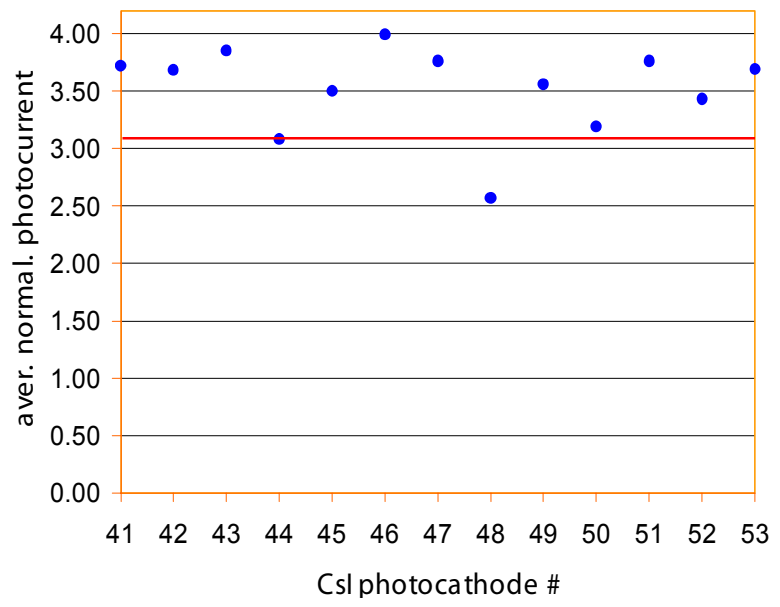

Fig. 7. Summary of the average normalized photocurrent of the first $13 \mathrm{CsI}$ PCs (41 to 53) of the series production. A possible acceptance threshold (red line) may be set at 3.10 . 
Module-1 has been equipped with old CsI PCs and tested in 2003. Module- 2 and Module-3 have been commissioned during summer ' 04 with final PCs (41 to 52) while Module-4 is presently under test. Fig. 8 shows Module-3 at the CERN/SPSX5 testbeam, fully equipped with electronics. A typical event, produced by $120 \mathrm{GeV} / \mathrm{c} \pi$ is shown in Fig. 9; the color coding represents the signal induced on the pads. Raw pad clusters originated by more than one detected Cherenkov photons, overlapping in the same pad or in neighboring pads, are split into smaller resolved clusters [2]. Fig. 10 presents a graph of the number of the resolved clusters per event in PC44 as a function of the gas gain, for several MIP positions. From this plot we can deduce a gain variation smaller than $5 \%$ and a PC response variation of about $10 \%$ over the scanned area.

Finally Fig. 11(a) and 11(b) show the summary of the performance, at an anode wires voltage of $2050 \mathrm{~V}$, of Module2 and Module-3, respectively. These data refer to a period of time of four weeks; since the number of clusters depends also on detector parameters, like the gas gain and the $\mathrm{C}_{6} \mathrm{~F}_{14} \mathrm{UV}$ transparency, which may change in time, a direct comparison of the PC performance can only be based on the CsI QE.

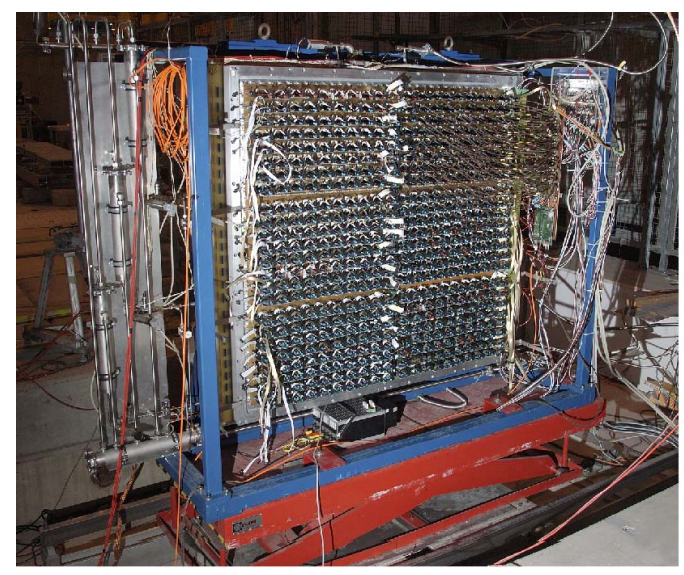

Fig. 8. A photo of Module-3 at the CERN/SPS-X5 testbeam.

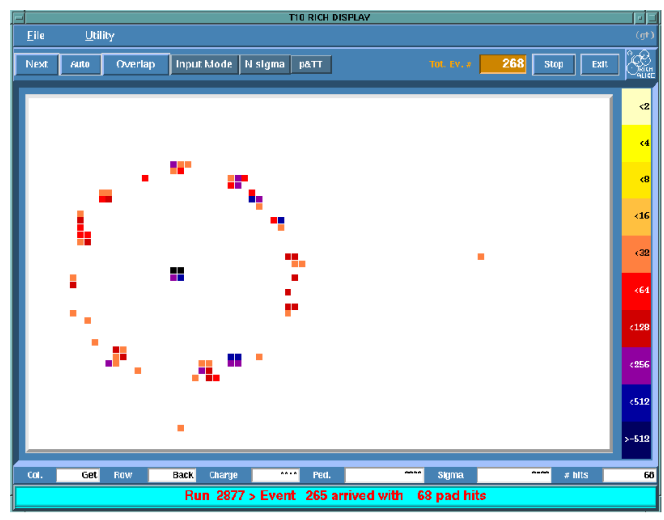

Fig. 9. An event produced by $120 \mathrm{GeV} / \mathrm{c} \pi$. The size of the panel corresponds to one CsI PC.

\section{CSI AGEING STUDIES}

The long term stability of CsI PCs kept under dry gas has been proven in the past [7], while it is as well known the CsI ageing resulting from irradiation with very high photon flux $\left(10^{12} \mathrm{~cm}^{-2} \mathrm{~s}^{-1}\right)$, very far from our application, or ions bombardment when operated in a gaseous detector [8]. CsI ageing has to be taken into account in the PCs validation for the series production, since the optimal PID performance should be kept during the 10 years operation of ALICE.

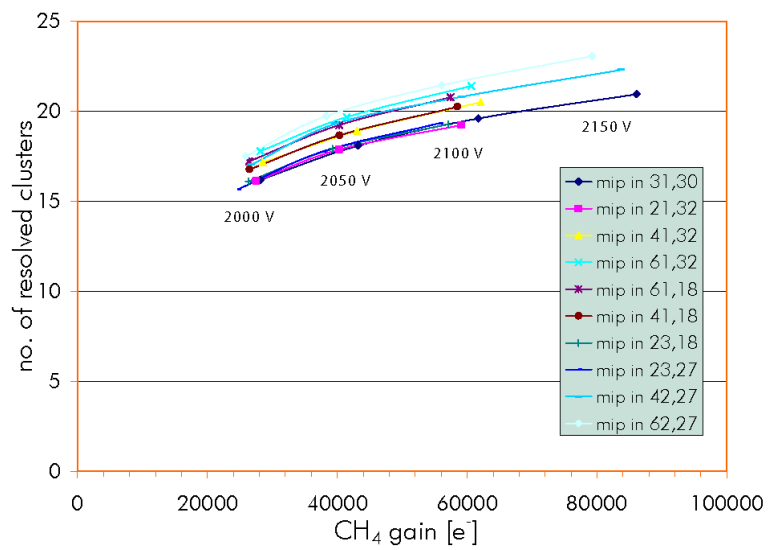

Fig. 10. The number of resolved clusters in PC44 as a function of the gas gain, in several MIP positions.
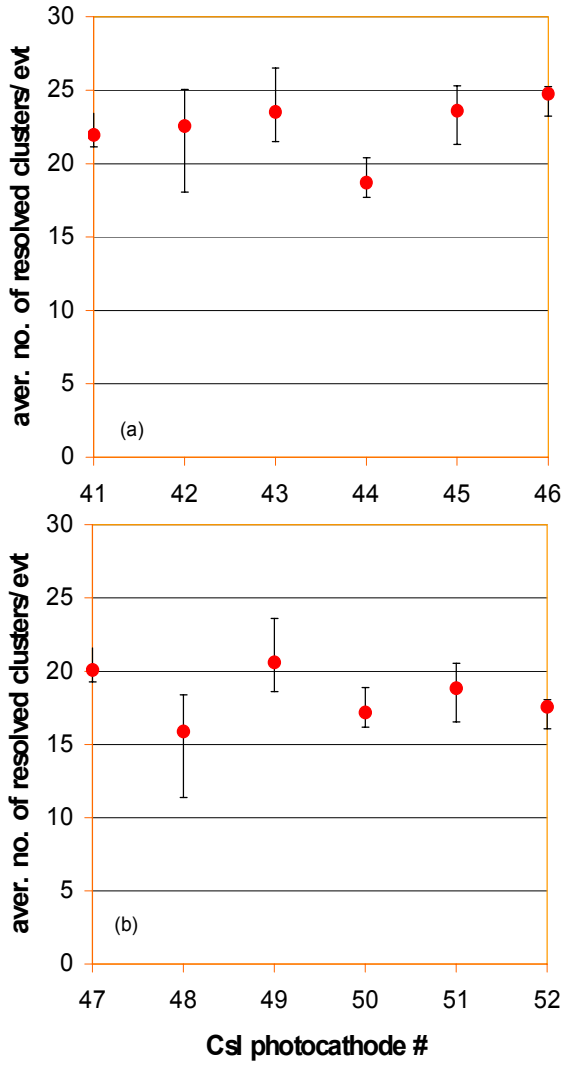

Fig. 11. The average number of resolved clusters for each PC in Module-2 (a) and Module-3 (b) at $2050 \mathrm{~V}$. The error bars represent the spread over the full $\mathrm{PC}$ area. 


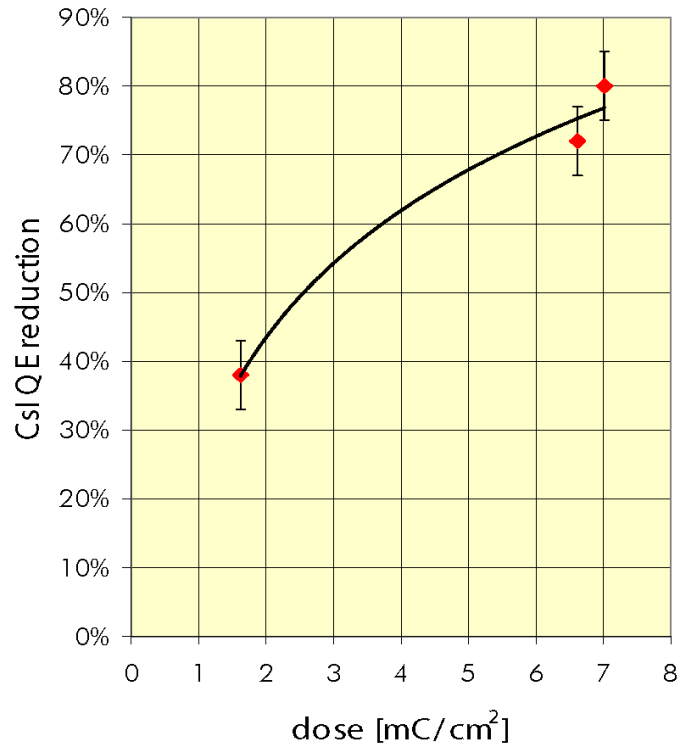

Fig. 12. The integral CsI QE reduction as a function of the accumulated dose on the PC (each value corresponds to a different irradiated spot).

Available data usually refer to small samples studied in accelerated ageing tests. We performed a test by irradiating with a collimated Sr-90 source a final size CsI PC in real operating conditions, namely installed in a detector prototype operated with $\mathrm{CH}_{4}$. Fig. 12 shows the CsI QE total reduction measured by the VUV scanner for three accumulated doses on different PC positions [9]. Given the observed non-linear behavior, new measurements are needed to study in more detail the low doses region, since the dose expected on the HMPID detectors in 10 years of ALICE operation amounts to $1 \mathrm{mC} / \mathrm{cm}^{2}$.

\section{SUMMARY AND FUTURE PLANS}

Five out of seven modules of the ALICE HMPID detector have been assembled; four modules have been tested with final
FEE. The CsI PCs mass production has started, 12 out of a total of 42 have been assembled and tested. Testbeam data of two modules equipped with final CsI PCs are being analyzed to establish the acceptance threshold on the QE for the series production. Further ageing studies are needed to include in the PC validation the needed margin for PID performance. The remaining modules will be equipped with final PCs and tested with cosmic rays before the installation in the ALICE magnet, foreseen in the period from January to April 2006.

\section{REFERENCES}

[1] ALICE collaboration, Technical Proposal, CERN/LHCC 95/71 and Physics Performance Report, CERN/LHCC 2003/49.

[2] ALICE collaboration, ALICE HMPID Technical Design Report, CERN/LHCC 98/19.

[3] J.C. Santiard, K. Marent, "The Gassiplex0.7-2 Integrated Front-End Analog Processor for the HMPID and the Dimuon Spectrometer of ALICE", Proceedings of the $5^{\text {th }}$ Workshop on Electronics for the LHC experiments, Snowmass, September 1999, p. 431.

[4] E. Schyns, "Status of large area CsI photocathode developments ", Nucl. Instr. And Meth. A494 (2002), 441; A. Braem, C. Joram, F. Piuz, E. Schyns and J. Séguinot, "Technology of photocathode production", Nucl. Instr. And Meth. A502 (2003), 205.

[5] H. Hoedlmoser, CERN, CH1211 Geneva 23, Switzerland, paper in preparation.

[6] A. Di Mauro, D. Cozza, M. Davenport, D. Di Bari, D. Elia, P. Martinengo, A. Morsch, E. Nappi, G. Paic, F. Piuz, "Performance of large area CsI-RICH prototypes for ALICE at LHC:", Nucl. Instr. And Meth. A433 (1999), 190.

[7] A. Di Mauro, P. Martinengo, F. Piuz, E. Schyns, J. van Beelen and T. D. Williams, "Study of the quantum efficiency of CsI photo-cathodes exposed to oxygen and water vapour", Nucl. Instr. And Meth.A461 (2001), 584; A. Braem, M. Davenport, A. Di Mauro, P. Martinengo, E. Nappi, G. Paic, F. Piuz, E. Schyns, "Aging of large area CsI photocathodes for the ALICE HMPID prototypes", Nucl. Instr. And Meth.A515 (2003), 307.

[8] A.Breskin, "CsI UV photocathodes: history and mistery", Nucl. Instr. And Meth.A371 (1996); J. Va'vra, "Photon detectors with gaseous amplification", Nucl. Instr. And Meth.A387 (1997), 137; J. Va'vra, A. Breskin, A. Buzulutskov, R. Chechik, E. Shefer, "Study of CsI photocathodes: volume resistivity and ageing", Nucl. Instr. And Meth.A387 (1997),154.

[9] F. Piuz, CERN, CH1211 Geneva 23, Switzerland, paper in preparation. 